\title{
La industria de la comunicación gráfica en Barranquilla, Colombia: Análisis sectorial desde el enfoque de las cinco fuerzas competitivas
}

\author{
The graphic communications industry in Barranquilla, Colombia: Sectoral based analysis of its \\ competitive five forces
}

D0I: http://dx.doi.org/10.21676/23897848.1683

Jahir Enrique Lombana-Coy Ph.D. Universidad del Norte, Barranquilla, Colombia. lombanaj@uninorte.edu.co

Alberto Mario Molina-Rocha MBA, Universidad del Norte, Barranquilla, Colombia. albermmolina@yahoo.com.co

Andrés Felipe Muñoz-Vergel MBA, Universidad del Norte, Barranquilla, Colombia. andmunozv@gmail.com

Jaime Alberto Muñoz-Vergel MBA, Universidad del Norte, Barranquilla, Colombia. jaimeac78@hotmail.com

Tipología: Artículo de Reflexión Fecha de Recibido: Enero 20 de 2016

Fecha de Aceptación: Marzo 10 de 2016

Para citar este artículo: Lombana, C. J., Molina, R. A., Muñoz, V. A. \& Muñoz, V. J. (2016). La industria de la comunicación gráfica en Barranquilla,

Colombia: análisis sectorial desde el enfoque de las cinco fuerzas competitivas.

Clío América. 10 (19), 73 - 89
Resumen: A través del modelo de las cinco fuerzas competitivas acuñadas por Michael Porter, se busca caracterizar e identificar los poderes competitivos de la industria de la comunicación gráfica en Barranquilla, Colombia. La información para el análisis es el resultado de entrevistas semi-estructuradas, realizadas a tomadores de decisión en grandes, medianas y pequeñas empresas, junto con información secundaria. Se concluye que el sector tiene muchos competidores en su interior, los proveedores son identificables y no son exclusivos, los clientes están atomizados, pero exigen productos a la medida, hay entrantes potenciales con pocas barreras de entrada, pero el conocimiento de la región y la diferenciación han servido hasta ahora para limitar su ingreso. Las empresas encuentran consenso en que los competidores en Barranquilla son fuertes, pero no hay un grado de asociatividad que les permita afrontar de manera conjunta los embates de la competencia. Es precisamente en este tema donde se deja abierta la puerta a investigaciones que promuevan la elaboración de un plan y direccionamiento sectorial.

Palabras clave: competitividad, cinco fuerzas, estrategia competitiva, comunicación gráfica.

JEL: L110, L220, M200

Abstract: Through the five competitive forces model of Michael Porter, this research seeks to characterize and identify the competitive power of the industry of graphic communications in Barranquilla-Colombia. The information for analysis is the result of semi-structured interviews conducted with decision makers in large, medium and small enterprises and secondary information. The main conclusions are that the sector has many competitors inside, suppliers are identifiable but not exclusive, customers are atomized but require customized products, there are potential entrants with low barriers to entry, however knowledge of the region and differentiation have served, so far, to limit their entrance. Companies find consensus that in Barranquilla, competitors are strong, but there is a lack of association that avoid them to jointly face the onslaught of competition. It is precisely in this area where the door is open to research promoting the development of a sectoral strategic plan.

Keywords: competitiveness, five forces, competitive strategy, graphic communication. 
La industria de la comunicación gráfica en Barranquilla, Colombia: Análisis sectorial desde el enfoque de las cinco fuerzas competitivas

\section{Introducción}

La industria de comunicación gráfica está presentando una tendencia creciente como negocio en Colombia y en particular en la ciudad de Barranquilla. Sin embargo, más allá de las mediciones gremiales e informes puntuales, no existe una caracterización enfocada hacia la formulación de estrategias sectoriales y mucho menos para su ejecución en un direccionamiento estratégico. Esta investigación tiene como objetivo caracterizar e identificar las fuerzas competitivas de la industria de la comunicación gráfica en Barranquilla para la pequeña, mediana y gran empresa (se excluye la microempresa) como base para la elaboración potencial de una formulación y direccionamiento estratégico sectorial. Para lograr este objetivo, el documento se divide en cuatro partes: en esta introducción se incluye una revisión del estado del arte en la investigación del sector en el país y el debate teórico sobre el modelo de las cinco fuerzas competitivas acuñadas por Michael Porter que es utilizado como marco en este estudio. Luego se incluye la metodología para a continuación realizar el análisis y resultados de las cinco fuerzas competitivas en la industria. La última parte, a manera de conclusión, presenta los relacionamientos de las cinco fuerzas competitivas aplicadas a la industria de la comunicación gráfica en Barranquilla.

\section{Estado del Arte de la Investigación en la Industria de la Comunicación Gráfica}

Los estudios relacionados sobre el desempeño sectorial de la industria de la comunicación gráfica son muy limitados en Colombia, sin embargo se pueden encontrar algunos realizados por el Programa de Transformación Productiva, como por ejemplo, el Informe Final del Sector de la Comunicación Gráfica, desarrollado por el Ministerio de Comercio, Industria y Turismo MinCIyT en el año (2008), donde se realiza un análisis de este sector a nivel mundial, luego se enfoca a nivel nacional, con el fin de mostrar estrategias y propuesta de valor para el desarrollo de este sector. Otro es el Informe de Sostenibilidad (MinCIyT, 2012) realizado así mismo por el Programa de Transformación Productiva PTP, el cual fue el primer informe de sostenibilidad de dicho sector, donde se presenta una visión general de la gestión, desarrollo y retos de las empresas en dicho año. Finalmente se encuentra un par de estudios sobre las formas de cooperación para formar aglomeraciones de empresa en el sector (Valero, 2004) y las competencias relevantes como estudio de caso en el sector (Medina, Delgado \& Lavado, 2012).

Por otra parte, en Colombia, resulta común encontrar investigaciones realizadas por entidades gubernamentales, tales como el DANE, con la Gran Encuesta Integrada de los Hogares (DANE, 2013), que muestra la empleabilidad por cada uno de los sectores, así como también los realizados por las diferentes agremiaciones que reúnen los sectores empresariales específicos. En este punto es importante mencionar a la Asociación Colombiana de la Industria de la Comunicación Gráfica (ANDIGRAF, 2013-2015), que emite boletines coyunturales y estudios en los cuales se pueden conocer los principales indicadores del sector objeto de estudio, como también los seminarios y proyectos del Centro de Desarrollo Tecnológico para la Competitividad de la Industria de la Comunicación Gráfica (CIGRAF) y del Centro para la Industria de la Comunicación Gráfica (CENIGRAF). En relación a los estudios mencionados anteriormente, se resalta que estos se hacen de forma general y a nivel nacional, mencionando aspectos relevantes, en algunos casos, a nivel regional y local. Sin embargo, no se detallan a ciudades específicas. Por lo anterior, un estudio como el planteado no tiene precedentes en el sector y es entonces que basados en una herramienta de análisis estratégico como el DOFA (Debilidades, Oportunidades, Fortalezas y Amenazas) es como se busca presentar el ambiente competitivo contexto en que surgen, se organizan y gestionan las empresas de la industria de comunicación gráfica en Barranquilla, Colombia (ver tabla 1). 
-Tabla 1.

\section{Matriz DOFA de la Industria de la Comunicación Gráfica en la Ciudad de Barranquilla}

\begin{tabular}{|c|c|c|c|}
\hline Debilidades & Fortalezas & Oportunidades & Amenazas \\
\hline \multicolumn{4}{|c|}{ Eslabón Primario } \\
\hline $\begin{array}{l}\text { Falta de estudios de caracteri- } \\
\text { zación de la industria a nivel } \\
\text { local. }\end{array}$ & $\begin{array}{l}\text { Disponibilidad de recursos para } \\
\text { afrontar nueva competencia. }\end{array}$ & $\begin{array}{c}\text { Facilidad para cambiar } \\
\text { proveedores. }\end{array}$ & $\begin{array}{l}\text { Apertura económica que } \\
\text { incentiva la llegada de nuevos } \\
\text { competidores. }\end{array}$ \\
\hline Costos fijos elevados. & $\begin{array}{l}\text { Innovación como respues- } \\
\text { ta a la entrada de nuevos } \\
\text { competidores. }\end{array}$ & $\begin{array}{l}\text { Poca concentración de } \\
\text { compradores. }\end{array}$ & $\begin{array}{c}\text { Compradores que son com- } \\
\text { petencia (integración hacia } \\
\text { adelante). }\end{array}$ \\
\hline $\begin{array}{c}\text { Alta dependencia de los } \\
\text { impresos. }\end{array}$ & $\begin{array}{c}\text { Know-how como principal ven- } \\
\text { taja competitiva. }\end{array}$ & $\begin{array}{c}\text { Alta dependencia de clientes } \\
\text { hacia la industria. }\end{array}$ & $\begin{array}{l}\text { Proveedores que son competen- } \\
\text { cia (integración hacia atrás). }\end{array}$ \\
\hline $\begin{array}{l}\text { Marcas poco posicionadas en el } \\
\text { mercado. }\end{array}$ & Canales de distribución propios. & $\begin{array}{c}\text { Reconocimiento en el exte- } \\
\text { rior de productos y servicios } \\
\text { nacionales. }\end{array}$ & Devaluación del dólar. \\
\hline $\begin{array}{l}\text { Poco conocimiento de potencia- } \\
\text { les competidores foráneos. }\end{array}$ & $\begin{array}{l}\text { Identificación de los principales } \\
\text { competidores locales. }\end{array}$ & & Ralentización de la economía. \\
\hline $\begin{array}{l}\text { Poca acción ante la aparición } \\
\text { de las redes sociales. }\end{array}$ & $\begin{array}{l}\text { Oferta de productos con alto } \\
\text { valor agregado. }\end{array}$ & & \\
\hline \multicolumn{4}{|l|}{$\begin{array}{l}\text { Falta de emprendimiento y } \\
\text { visión tendientes a la incursión } \\
\text { en mercados internacionales. }\end{array}$} \\
\hline \multicolumn{4}{|c|}{ Cadenas } \\
\hline Alta informalidad. & Poca intermediación. & $\begin{array}{l}\text { Programa de Transformación } \\
\text { Productiva PTP - MinCIT }\end{array}$ & $\begin{array}{l}\text { Poca articulación Gobierno- } \\
\text { Instituciones- Empresas. }\end{array}$ \\
\hline $\begin{array}{l}\text { Bajos márgenes de } \\
\text { comercialización. }\end{array}$ & $\begin{array}{c}\text { Institucionalidad representada } \\
\text { en ANDIGRAF - SENA. }\end{array}$ & & \\
\hline \multicolumn{4}{|c|}{ Mercados } \\
\hline $\begin{array}{c}\text { Brecha competitiva con países } \\
\text { líderes de la industria. }\end{array}$ & $\begin{array}{l}\text { Conocimiento del mercado y } \\
\text { cultura local. }\end{array}$ & Industria en crecimiento. & $\begin{array}{l}\text { Entrada de las redes sociales } \\
\text { como producto sustituto. }\end{array}$ \\
\hline \multirow[t]{2}{*}{$\begin{array}{l}\text { Precios presionados a la baja } \\
\text { por alta oferta e informalidad. }\end{array}$} & $\begin{array}{l}\text { Demanda creciente de produc- } \\
\text { tos personalizados. }\end{array}$ & $\begin{array}{l}\text { Acuerdos comerciales que } \\
\text { permiten entrada a nuevos } \\
\text { mercados internacionales. }\end{array}$ & Competencia global. \\
\hline & & $\begin{array}{l}\text { Llegada de nuevas empresas al } \\
\text { país potenciales clientes. }\end{array}$ & Estacionalidad de la demanda. \\
\hline \multicolumn{4}{|c|}{ Entorno Normativo } \\
\hline $\begin{array}{c}\text { Desconocimiento de programas } \\
\text { como el PTP. }\end{array}$ & $\begin{array}{c}\text { Existencia de Programas como } \\
\text { el PTP. }\end{array}$ & Reingeniería de la Industria. & $\begin{array}{l}\text { Inefectividad de políticas } \\
\text { públicas que incentivan a la } \\
\text { industria- }\end{array}$ \\
\hline
\end{tabular}

Fuente: Elaboración propia 
La industria de la comunicación gráfica en Barranquilla, Colombia: Análisis sectorial desde el enfoque de las cinco fuerzas competitivas

\section{Enfoque crítico al modelo teórico de las cinco fuerzas competitivas}

El modelo de Porter $(1979,2008)$ de las cinco fuerzas competitivas ha sido ampliamente difundido en el mundo académico y aplicado en la práctica como enfoque explicativo de la estrategia competitiva de los negocios. Su enfoque pragmático desde el mundo académico ha hecho que su adopción en las empresas se vea como un quehacer normal en su análisis estratégico (Brandenburger, 2002). La rivalidad de la competencia actual, el poder de negociación de las empresas de un sector frente a sus proveedores o sus compradores, la adición de los productos sustitutos y de los potenciales entrantes configuran en conjunto las cinco fuerzas competitivas que definen la estrategia competitiva de la industria. De hecho, el modelo de las cinco fuerzas competitivas enfrenta poderes de negociación, valor, costos de oportunidad y por supuesto escala de los participantes que se enfrentan de manera que se pueden explicar con teoría de juegos (Branderburger \& Stuart, 1996).

Existen, eso sí, autores que plantean del modelo de Porter lo limitado que puede ser en contextos de países en desarrollo. Hoskisson, Eden, Lau y Wright (2000) y Wright, Filatotchev, Hoskisson y Peng (2005) hacen precisamente un recorrido sobre la investigación en estrategia empresarial para países en desarrollo. Narayanan y Fahey (2005) enuncian que las estrategias en economías emergentes como el contexto institucional, no necesariamente son aplicables en el modelo de las cinco fuerzas competitivas, principalmente porque el supuesto de libre comercio no es el prevaleciente y particularmente la participación del gobierno en ciertos contextos puede ser muy relevante. Esto ya se había percibido en el génesis de las cinco fuerzas competitivas con Dunning (1993) y Rugman y Verbeke (1993) quienes aducían que los países particularmente emergentes, hacían que las empresas modificaran sus estrategias para adecuarse a los propios contextos, quedando en evidencia la percepción de Porter hacia un mundo totalmente liberal, particularmente en sectores de interés para EE. UU. Ejemplos empíricos como Nelson (1990) en Brasil y Peng y Luo (2000) en China, plantean precisamente que conceptos de estrategia competitiva provenientes del mainstream, no eran necesariamente aplicables a países en desarrollo.

Otro elemento a considerar en las cinco fuerzas competitivas es la unidad de análisis que pretende explicar. Para McGahan y Porter (1997), es claro que la industria es quien gobierna la estrategia competitiva de las empresas individuales. En contravía sobre las consideraciones de que la unidad de análisis para conformar la estrategia debe ser la misma empresa como lo anotaba Wernerfelt (1984) con la perspectiva del enfoque basado en recursos y que posteriormente fue enriquecido con Barney (1991) y Peteraf (1993). Porter (1996) hace algunos reparos a sus críticos, principalmente haciendo distinción entre estrategia competitiva y efectividad operacional, siendo la primera referida a opciones y la segunda a referentes, mejores prácticas y minimización de costos. Respecto a la unidad de análisis, su enfoque micro lo dirige necesariamente a una relación directa con las empresas, pero continua su discurso haciendo énfasis en la industria, particularmente acuñando el concepto de clúster como conjunto de empresas que cooperan y rivalizan en un espacio geográfico determinado. Finalmente, y respecto a las otras fuerzas que sus críticos mencionan como necesarias en el modelo (gobierno e internet), él las analiza como exógenas y se refiere a ellas como determinantes que facilitan o dificultan las estrategias de las empresas dentro de la industria. Con este marco en mente se atribuye a la funcionalidad del modelo su aplicación, y es precisamente esa la razón para utilizarlo en este sector.

\section{Metodología}

El estudio es de carácter descriptivo, donde se especifican propiedades y características de un sector. Para esto se utiliza el enfoque de las cinco fuerzas competitivas propuesto por Porter (1979 y 2008) a partir de información extraída de encuestas y entrevistas a profundidad a empresarios de la industria de comunicación gráfica en Barranquilla.

Para delimitar el universo de empresas, se referenciaron 422 empresas registradas en la Cámara de Comercio de Barranquilla, de las cuales 52 están 
ubicadas en municipios aledaños (Baranoa, Galapa, Luruaco, Malambo, Puerto Colombia, Santo Tomas, Sabanalarga y Soledad). Así, de las restantes 370 ubicadas efectivamente, hay 152 registrados como Personas Naturales y 258 como Personas Jurídicas. Dentro de las personas naturales (152), son: 1 empresa pequeña y 151 microempresas; y dentro de las Personas Jurídicas (258) de acuerdo al nivel de activos, son: 235 microempresas y 23 empresas pequeña, mediana y grandes empresas, estas últimas en las que se centra este estudio. Adicional a las 23 y acorde con información suministrada por la ANDIGRAF se incluyen las principales empresas en ventas de la ciudad de Barranquilla para el año 2013 y 2014 (12 empresas). La población seleccionada fue de 35 empresas, de ellas 15 accedieron a participar en la encuesta y en una entrevista semiestructurada que busca recoger la información para caracterizar la industria de la comunicación gráfica a través del modelo de las cinco fuerzas competitivas.

\section{Resultados}

Con el fin de establecer un marco que permita determinar la estructura de la industria de la comunicación gráfica en la ciudad de Barranquilla, en esta sección se hace un análisis de cada una de las fuerzas competitivas según Porter.

\section{Fuerza 1: Rivalidad entre competidores}

En esta fuerza se permite indagar si es preciso mejorar 0 rediseñar las estrategias competitivas existentes, en la medida que el análisis obliga a comparar las ventajas competitivas con los otros rivales en un marco sectorial.

La tendencia, diversidad y tamaño de la industria determinan en gran medida que un sector puede soportar los embates estratégicos de sus proveedores y compradores. Asimismo, su estructura de costos y la diversidad en productos es clave para enfrentar las fuerzas de posibles entrantes y productos sustitutos.

\section{a) Crecimiento de la industria}

El $66.6 \%$ considera que el crecimiento experimentado por la industria hasta 2015 ha sido alto, y se espera siga así a pesar de las condiciones macroeconómicas en que se encuentra el país. La expectativa de crecimiento en particular de la industria de la comunicación gráfica de Barranquilla está motivada por el auge turístico, cultural, empresarial, comercial e inmobiliario que vive la ciudad, la cual se presenta como unos de los destinos de inversión más atractivos del país y del Caribe. Todo lo anterior incentiva la llegada de nuevos competidores atraídos por las condiciones antes mencionadas y motivados con hacerse a una porción de un mercado que todavía no está saturado y que presenta grandes oportunidades crecimiento.

\section{b) Diversidad de competidores}

En la figura 1 se observa que los entrevistados identificaron a más de 10 competidores, mostrando además que, varias empresas coinciden en identificar un mismo competidor. Se puede observar que Digiprint es la empresa que mayor número de veces fue mencionado como competidor, seguida de Dolmen y Ditar, las cuales, por nivel de venta, son también las más grandes del sector. En el caso particular de Digiprint se puede decir que es una empresa que se destaca por tener una red de contactos muy amplia y fuerte, permitiéndole desarrollar de manera efectiva una base de clientes extensa, y considerada por las demás como una de las competidoras más fuertes del mercado. Lo anterior pone de manifiesto una variedad de competidores, que se desenvuelven en cada uno de los segmentos de esta industria, con diferencias en sus objetivos, costos y estrategias competitivas dentro de un entorno totalmente heterogéneo.

\section{c) Condiciones de los costos}

La estructura de costos confirma que más del $60 \%$ de las empresas encuestadas presenta una proporción más alta de costos de fijos que de variables. Entre los costos más importantes se destacan los gastos de nómina y los servicios públicos. Así mismo, frente a una competencia de precios, las empresas deberán disminuir de manera representativa los costos fijos, para poder ser competitivos. Vale resaltar que, desde el punto de vista financiero, esta situación pone a las empresas de la industria en una posición de riesgo, debido a que, en la práctica, la reducción de costos fijos se dificulta. 


\section{- Figura 1.}

\section{Competidores Identificados por Empresa (\%)}

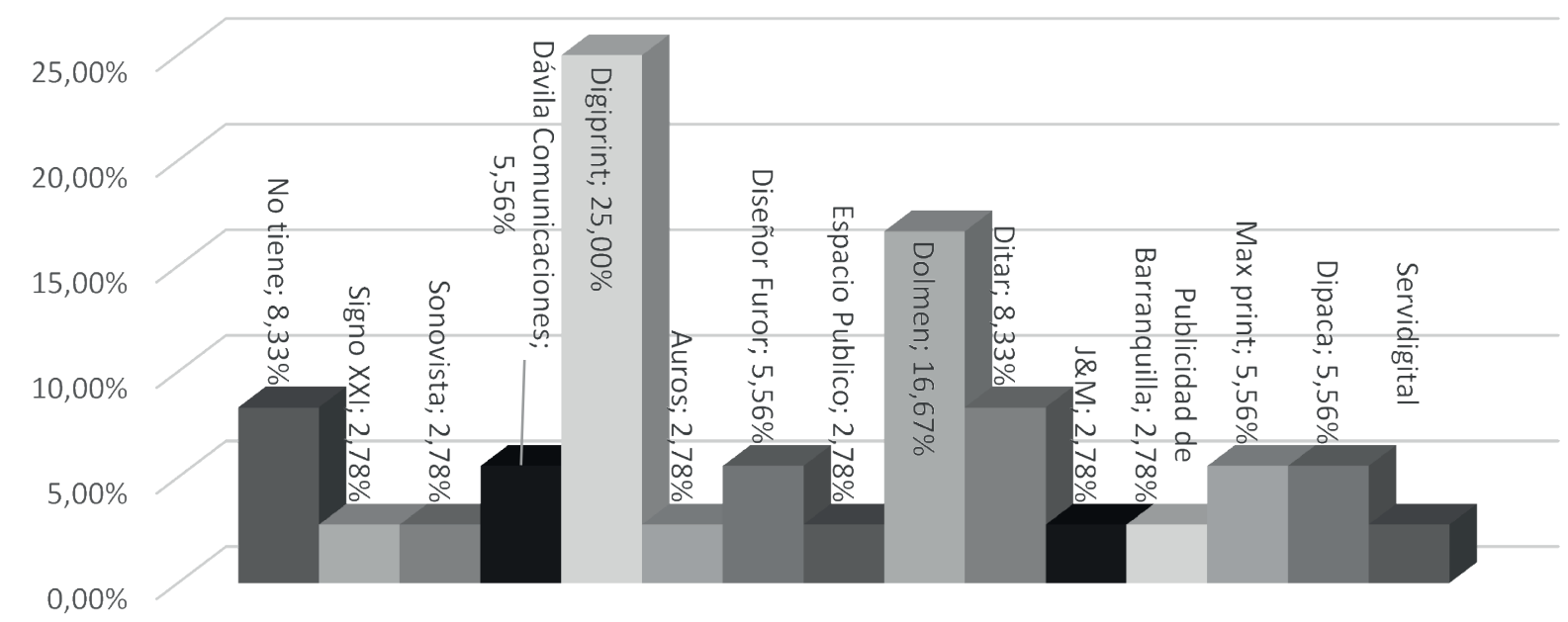

Fuente: Elaboración propia.

\section{d) Grandes aumentos de capacidad}

Este factor permite conocer la perspectiva de crecimiento de los empresarios de la industria. Según los resultados obtenidos, el $80 \%$ de los encuestados piensa aumentar la capacidad de producción para aumentar sus niveles de producción, al tiempo que fortalece su productividad. Estos aumentos de capacidad van de la mano con los factores que influyen en la perspectiva de crecimiento analizados anteriormente.

\section{e) Productos homogéneos}

Con esto se permite identificar, la variedad de productos que desarrolla cada una de las industrias, a pesar de estar, en ocasiones, en distintos segmentos, y por ende no competir en el mismo nicho de mercado. Según los encuestados, se puede observar en la tabla 2, que existen tres productos, considerados, los más importantes para la industria, y así mismo, para cada uno de los segmentos: impresos con un $66.67 \%$, avisos con un $26.67 \%$ y gran formato $26,67 \%$. De igual forma, las cajas y empaques ocupan un cuarto lugar destacándose del resto con un $6.67 \%$.

\section{Principales productos de la industria de comunicación gráfica en Barranquilla, Colombia}

Productos Participación en respuestas (\%)

Impresos 33,3

Avisos 13,3

Gran formato 13,3

Cajas 6,6

Diseño de páginas web 3,3

Dron 3,3

Artes 3,3

Planos 3,3

Flyers 3,3

Vallas 3,3

Etiquetas 3,3

Muebles 3,3

Fuente: Elaboración propia. 


\section{f) Ciclo de vida}

Desde la perspectiva de los empresarios encuestados, el $46.67 \%$ señala que la etapa en cual se encuentra su empresa es madurez, esto se debe a la antigüedad y experticia que llevan desarrollando su actividad. Por su parte, otro $40 \%$ considera que la industria está en crecimiento y el restante 13\% se distribuyen entre empresas que consideran a la industria en nacimiento o declive.

Por otra parte, si se toma el análisis de la industria, los empresarios consideran que esta se encuentra en crecimiento $(80 \%)$, pues la mayoría de las empresas se encuentran desarrollando actividades estratégicas que les permitan crecer en sus márgenes de rentabilidad y sacarles ventaja a sus competidores.

\section{Fuerza 2: Poder de negociación de los proveedores}

Los proveedores pueden tener la intensión de integrarse hacia adelante si tienen el estímulo suficiente $y$ las condiciones del entorno empresarial se lo permiten, ello está dado en mayor medida de acuerdo a su grado de concentración y/o exclusividad en las materias primas que provean.

El $93.33 \%$ de los encuestados dijo conocer directamente a sus proveedores (entre los principales insumos que se surten de proveedores se encuentran papel, tintas, maquinaria y equipos e incluso software), es decir que hay un conocimiento de la cadena de abastecimiento hacia atrás, esto es importante en la medida que las empresas de la industria pueden realizar la planeación de su proceso de suministros y abastecimiento con el objetivo de poner a disposición de las plantas de producción los insumos o materias primas en las cantidades, condiciones y tiempos requeridos para la satisfacción de la demanda; por lo tanto, el conocimiento de sus proveedores se traduce en ventaja competitiva. El resto no registra información o no quisieron brindarla. Cabe anotar que, en algunos entrevistados hay cierto recelo a la hora de dar la información y expresaron que uno de sus mayores insumos para tener ventaja competitiva eran el conocimiento y la creatividad al servicio de la empresa.

\section{a)Concentración de proveedores}

De acuerdo a la encuesta (ver figura 2) se pudo evidenciar que los empresarios nombraron una cantidad importante de proveedores (19 en total), de estos, 17 son nacionales. En este sentido se puede decir que su poder de negociación es relativamente bajo al haber un abanico amplio de empresas ofreciendo insumos o materias primas que son poco diferenciadas. Las empresas encuestadas que expresaron tener solo proveedores nacionales, lo justificaban con la alta volatilidad del dólar y los riesgos externos no medibles.

El proveedor nacional más reconocidos por la industria fue Dispapeles, empresa líder con más de 45 años en el mercado, que comercializa productos de clase mundial y cuenta con la planta de producción más moderna de Colombia, donde se elaboran productos con alto valor agregado. A nivel regional la más destacada es Papelplot, empresa Barranquillera con más de 10 años en el mercado que se ha venido destacando y ganando el reconocimiento del mercado por la calidad de sus productos y servicios.

En el caso de los proveedores extranjeros los encuestados nombraron dos, Trinity Design (EE. UU.) y Susano (Brasil), en este caso, el poder de negociación de estos proveedores es alto debido a la especialización y al valor agregado de los productos y servicios que ofrecen a sus compradores colombianos, los cuales no ven tan fácil el cambio de estos proveedores debido al lastre competitivo que esto les puede causar. La empresa E-mage Consultig Group, por citar un ejemplo, expresa que el servicio en soporte y asesoría integral en el tema logístico para la realización de eventos en la ciudad de Miami, Florida, que le presta Trinity Design, es fundamental para lograr los objetivos propuestos en lo que respecta a ruedas de negocios y campañas publicitarias que necesiten sus compradores colombianos en Estados Unidos y los locales en la Florida. Entonces en este caso Trinity tiene un alto poder de negociación frente E-mage, quien considera a su proveedor como un aliado estratégico. Por otra parte, Susano suministra papel de alta calidad que no se produce en el país y que puede entrar precisamente en el análisis de exclusividad del siguiente aparte. 


\section{b) Exclusividad de los proveedores}

El 53.33\% de los encuestados expresa que los proveedores son exclusivos de la industria. Este aspecto es importante ya que la no exclusividad garantiza el suministro de insumos que son primordiales para el funcionamiento y competitividad de la industria. Por su parte, la exclusividad, desde el punto de vista de los proveedores, les crea una dependencia que puede ser analizada a la hora de ver el análisis estratégico entre los dos eslabones de la cadena.
En la industria se presenta cierta tendencia de integración hacia adelante (ver figura 3), donde algunos proveedores se convierten en competidores, el $46 \%$ de los entrevistados los considera potencialmente. Hay una motivación para los proveedores de tener relación directa con los consumidores finales, manejando anales de distribución, fijando precios de producto final generando mayor rentabilidad a su operación, siendo también considerado por los encuestados, que los grandes márgenes los obtienen los intermediarios que atienden la demanda final de productos y servicios.

\section{Figura 2.}

\section{Principales Proveedores}

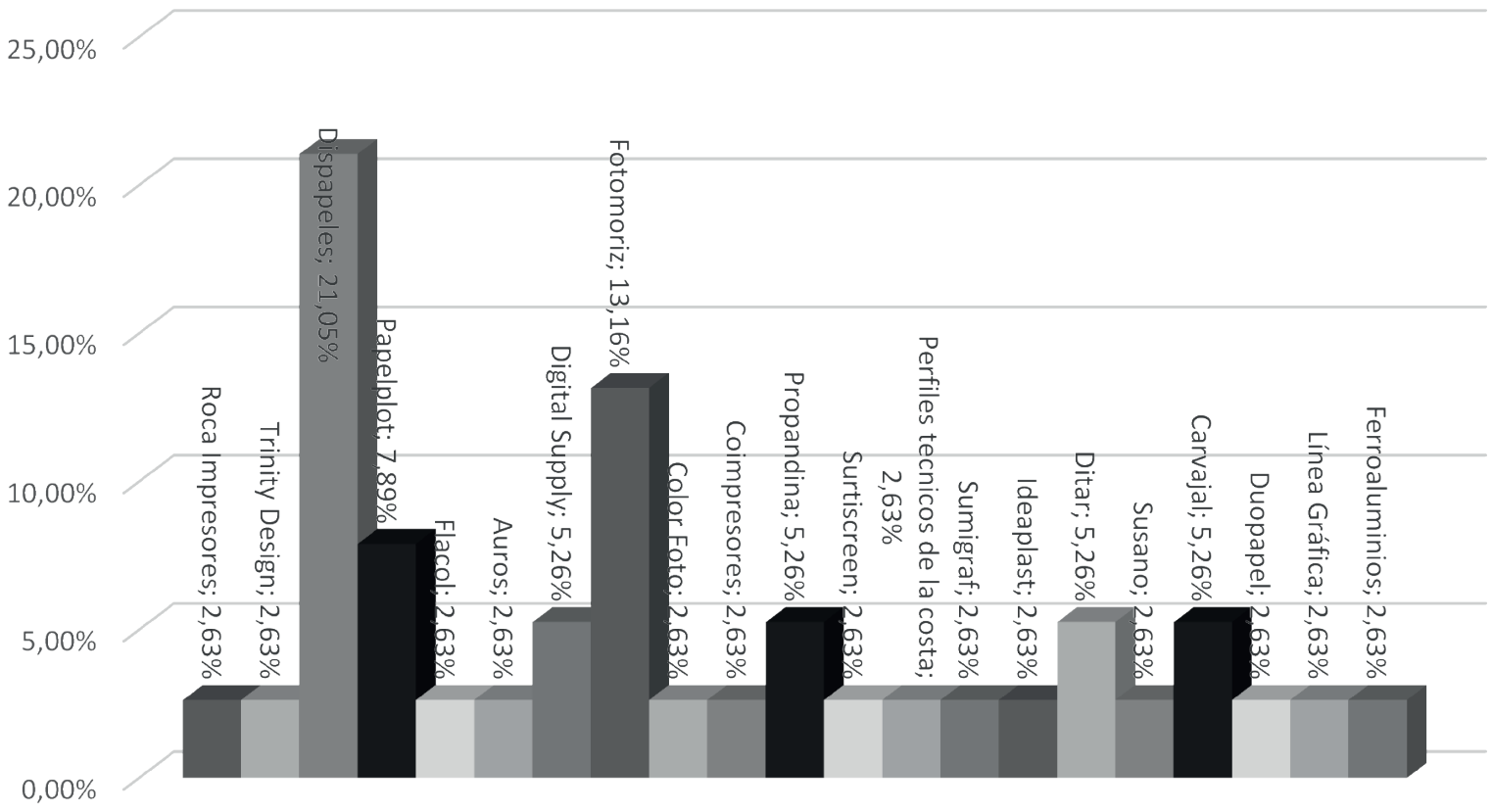

Fuente: Elaboración propia. 
Figura 3.

\section{Principales Proveedores Considerados Competidores}

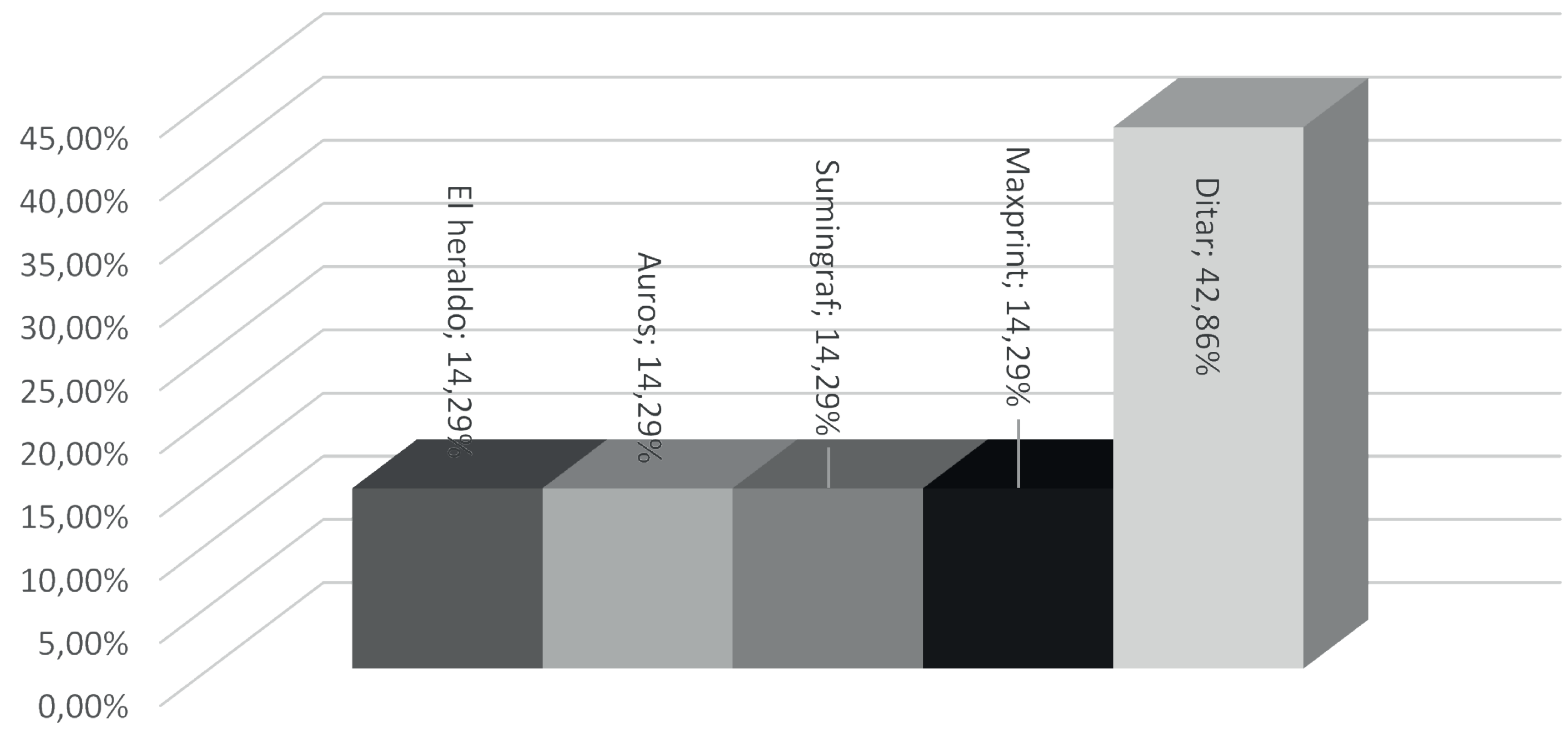

Fuente: Elaboración propia.

\section{Fuerza 3: Poder de negociación de los compradores}

Los compradores en su cadena de suministro tienen la capacidad de afectar a sus proveedores. El relacionamiento entre los eslabones de la cadena, la sensibilidad al precio, el grado de concentración, dependencia y escala de los agentes económicos y los aspectos cualitativos determinan la fortaleza y poder de negociación de los compradores.

\section{a) Forma de venta}

Las empresas venden sus productos al consumidor final, lo cual le permite al comprador obtener un mejor precio al ubicarse estratégicamente en la cadena de valor. Solamente el $11.76 \%$ de las empresas utilizan, en ocasiones, intermediarios (Efecty Medio y J\&M), que puede encarecer el precio 0 , en procura de mantenerlo reducen la rentabilidad de la empresa.

\section{b)Información acerca del proveedor}

La facilidad que tienen las empresas para cambiar proveedores, denota información precisa para los compradores sobre la existencia de muchos proveedores en el sector. El $66.67 \%$ de las empresas consideran que le resulta fácil cambiar un proveedor, por tanto, los compradores aumentarán su capacidad de negociación ya que tienen más posibilidad de cambiar de proveedor de mayor y mejor calidad. Lo anterior se evidenció aún más al momento de realizar las entrevistas por el complicado periodo económico de coyuntura, con una devaluación anual del peso acumulada al $35 \%$, donde muchas empresas manifestaron dejar de importar para comprar productos locales.

\section{c) Concentración de compradores}

Frente a este factor, los resultados de la encuesta denotan que existe tendencia a la atomización de los compradores, en la medida que un gran porcentaje de compradores tienen capacidad para comprar una porción importante, sino toda la producción de la industria $67 \%$ cree que hay muchos compradores. Esto incide de manera directa en el poder de negociación, ya que los compradores, por no estar cohesionados, pierden la capacidad de exigir y las empresas pueden dejar ir a un comprador sin riesgo de sentirse, porque 
esto afectaría significativamente su nivel de ventas y por ende la rentabilidad de la empresa.

\section{d) Dependencia}

El nivel de dependencia de los compradores hacia la industria hace que estos varíen su poder de negociación frente a los proveedores. De acuerdo a los resultados obtenidos, existe una alta dependencia de los compradores hacia los productos de esta industria $y$, según lo manifestaron, pueden incidir de forma importante. En concordancia con lo anterior, en la figura 4 se muestran los compradores con mayor dependencia de la industria.

El sector de bebidas y alimentos, los partidos políticos, los eventos culturales y el sector comercial son los de mayor dependencia de esta industria, al punto que gran parte de sus ingresos y por ende de su éxito, está fundamentado en los productos o servicios que entrega la industria de la comunicación gráfica. Con respecto a los políticos, es importante anotar que estas ventas son estacionarias y presentan picos muy altos durante las elecciones de cada uno de los órganos que conforman el gobierno. Existen compradores coyunturales, que también tienen una alta dependencia de este sector, ya que la mayoría de sus inversiones se realizan en productos ofrecidos por empresas de esta industria, acorde con el nivel meta analizado anteriormente. En conclusión, el poder de negociación de estos compradores hacia la industria es bajo debido a la dependencia demostrada.

\section{Figura 4.}

\section{Compradores con mayor dependencia}

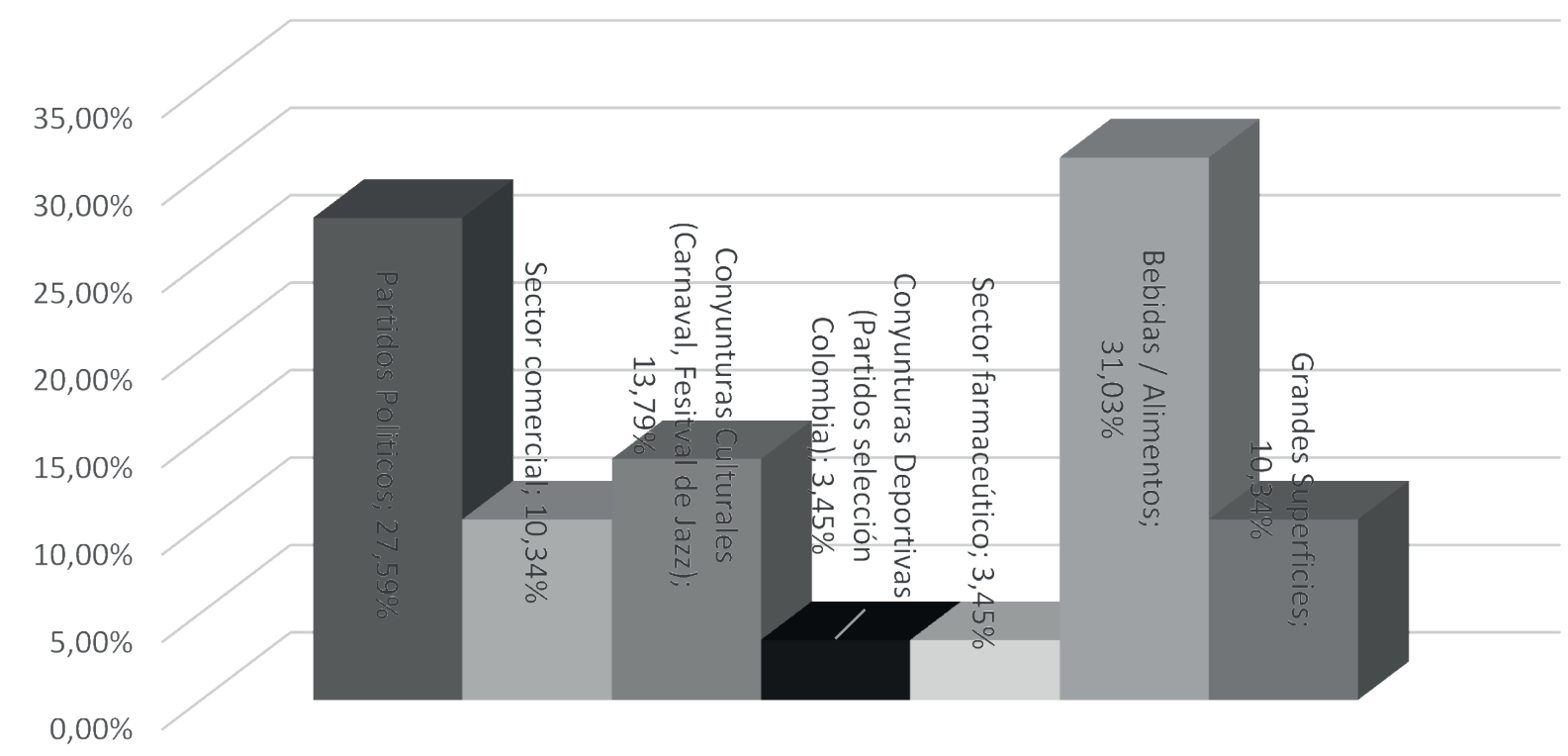

Fuente: Elaboración propia.

\section{e) Diferenciación}

Este factor permite conocer si los compradores exigen productos estandarizados por parte de las empresas del sector. De acuerdo a los resultados, de forma contundente se confirma que los productos exigidos por los compradores son diferenciados y con alto valor agregado, con tendencia a la personalización. Los encuestados tienen la flexibilidad para hacer productos a la medida por lo que realmente 
no es una ventaja para los compradores al exigir diseño y calidad específicos.

\section{f) Integración hacia atrás}

En la industria objeto de investigación, según la mayoría de las empresas encuestadas, coinciden en que existen compradores $(67 \%)$ que a la vez son competidores pues en ocasiones actúan como intermediarios frente al consumidor final y supone, que estos compradores son altamente sensibles al precio, ya que su rentabilidad o réditos serán el margen de intermediación.

\section{Fuerza 4: Posibles Entrantes}

Las empresas de la industria pueden crear barreras de entrada a potenciales competidores (p.ej.: agremiaciones cerradas, fidelización), ejercer presión para que institucionalmente los gobiernos incluyan obstáculos de ingreso (p.ej.: impuestos o normas) 0 incluir actividades que, al interior de la rivalidad existente entre empresa, la industria se fortalezca y evite ingresar nuevos entrantes. El mismo entorno puede limitar la entrada de competidores potenciales cuando, por ejemplo, el mercado está saturado.

\section{a) Posibles Entrantes Foráneos}

Existe una alta probabilidad de que haya empresas que estén interesadas en ingresar con sus negocios la ciudad de Barranquilla (ver figura 5), incluso algunas ya han llegado a participar del mercado local.

En este aparte es importante recalcar que la entidad que representa al gremio de la industria de la comunicación gráfica debe ponerse en la tarea de impulsar iniciativas para colocar barreras de entrada efectivas que limiten la entrada de nuevos competidores, si el fin es proteger al sector de la competencia externa.

JFigura 5.

\section{Posibles entrantes foráneos (otra ciudad)}

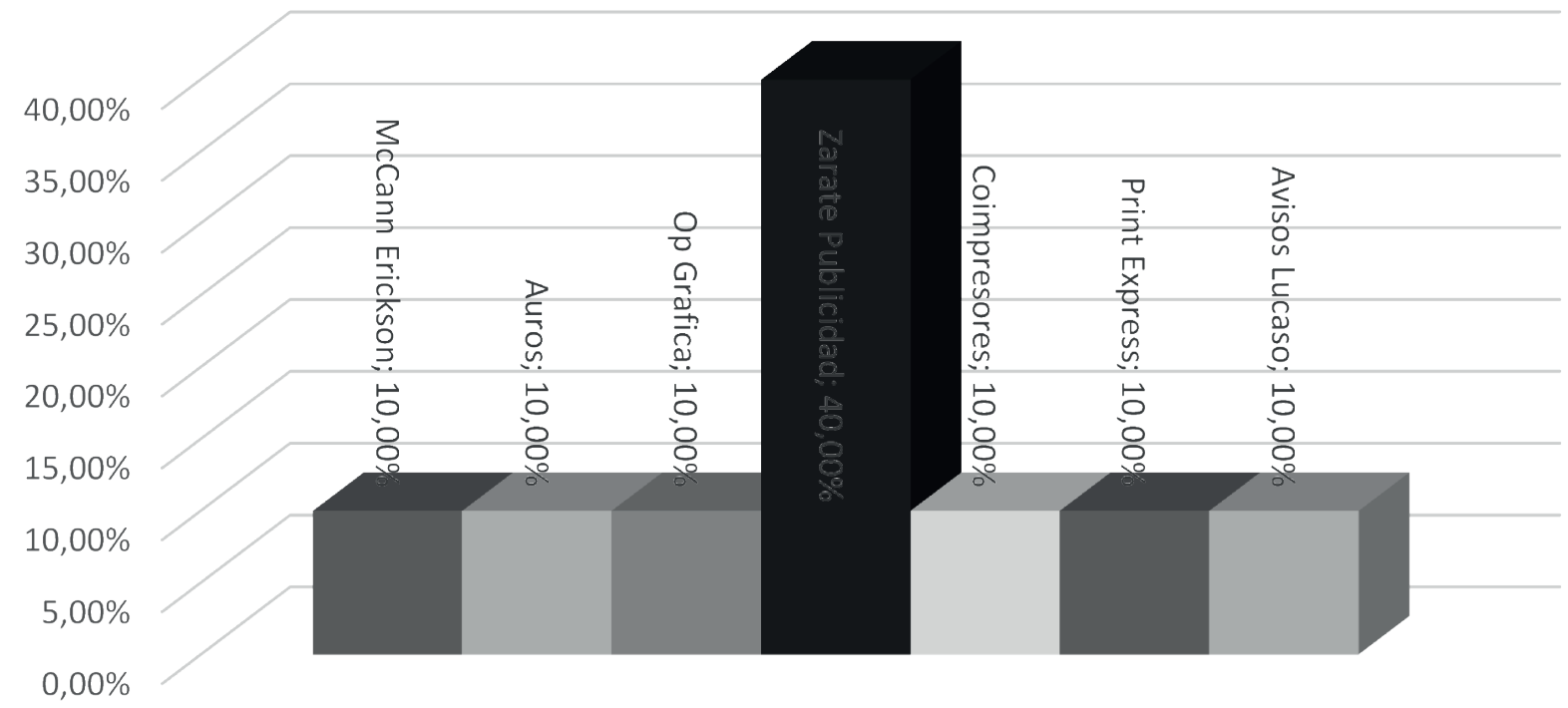

Fuente: Elaboración propia.

b)Inversión necesaria o requisitos de capital

A las empresas encuestadas se les consultó si se requiere un músculo financiero grande para entrar a la industria, y los resultados arrojan que el 93\% considera que se requiere de una cantidad importante de recursos económicos para poder ingresar a este sector. 
La industria de la comunicación gráfica en Barranquilla, Colombia: Análisis sectorial desde el enfoque de las cinco fuerzas competitivas

Entre las consideraciones que se tienen para esta apreciación, se mencionan como requisitos a una competencia legal y legítima:

i. Empresas formales, esto es debidamente constituidas y que su funcionamiento este acorde a las disposiciones legales y marcos regulatorios

ii. Equipos o maquinaria, comprados legalmente y conforme a lo dispuesto por ley

iii. Capital social fuerte para poder participar en licitaciones con distintas empresas del sector privado y público

Lo anterior toma especial relevancia al revisar el número de empresas ubicadas en este sector, ya que gran parte de estas son consideradas pequeñas empresas, y en su mayoría operan desde la informalidad y sin estándares de calidad que permitan fortalecer al sector. Es importante anotar que, si bien las empresas encuestadas, tienen concentrados sus compradores en corporativos, los comerciantes minoritarios diariamente no le permiten a las medianas y grandes empresas llegar a más consumidores, ya que, en ocasiones desde la informalidad, o desde la carencia de un estudio de costos, logran precios muy por debajo de los estándares del sector.

\section{c) Curva de experiencia}

Este factor hace referencia a las ventajas competitivas de las empresas del sector, las cuales han sido reforzadas por el transcurso del tiempo, así como también durante su curva de aprendizaje y que les permite tener una relación muy fuerte, de forma que logran una gran fidelización con sus compradores y que, por lo tanto, son barreras de entrada para nuevos competidores. El $71 \%$ de las empresas señalan el know how como su principal ventaja competitiva, ya que abarca toda la experiencia de una empresa en el desarrollo de una actividad durante un período de tiempo prolongado, en acciones propias de la gestión empresarial, tales como la tecnología de productos, procesos, entre otros. La ubicación privilegiada de las empresas en la ciudad de Barranquilla, se convierten en también en ventaja $14.29 \%$ que puede agregarse al acceso a insumos $9.52 \%$ y beneficios legales $4.76 \%$ como principales factores de competitividad creada.

\section{d) Barreras Gubernamentales}

Conforme a los resultados obtenidos, no se observan barreras gubernamentales fuertes que supongan costos importantes para la entrada de nuevos competidores, al tiempo que dificulten el ingreso de los mismos al sector. Tan sólo el $6.67 \%$ menciona que sí conocen políticas públicas específicas para el sector, sin embargo, la política en cuestión trata de un incentivo arancelario puntual, más no de protección integral o estructural a la industria, lo que denota una oportunidad para competidores foráneos y una amenaza para las empresas del sector que no cuentan con medidas que les permitan obtener por parte del gobierno central beneficios para el desarrollo de sus actividades. De igual forma, los empresarios de la ciudad concordaron en que el Gobierno debería tomar medidas en aspectos claves como la informalidad y la ilegalidad.

\section{e) Diferenciación del producto}

En relación a la diferenciación de los productos, este factor hace referencia al grado en que los consumidores distinguen un producto de otro; lo anterior basado en atributos propios de la marca o de la empresa que hace dificil que un posible entrante pueda competir con empresas ya asentadas en la ciudad. Según los resultados, no existe una tendencia clara y contundente que permita determinar si la marca o productos de las empresas existentes son barrera de entrada para nuevos competidores, sin embargo, la mayoría de los encuestados manifiestan que en conjunto (marca y producto) son obstáculos implícitos para nuevos entrantes. Desde el punto de vista de la escala y la fidelización de los compradores, los potenciales entrantes deben tener un músculo financiero para su rápido establecimiento y expansión.

\section{f) Acceso a canales de distribución}

Este factor hace referencia a la posibilidad para el potencial competidor de acceder a canales de distribución existentes, ya que, al complicarse la opción de pertenecer a estos, se dificulta que el consumidor final tenga posibilidad de adquirir el producto. Frente a lo anterior, es importante mencionar que la totalidad de las empresas de la 
industria cuenta con canal de distribución propio, lo cual permite asegurar que los productos lleguen al consumidor final en las condiciones establecidas por ellos. Así mismo, se puede evidenciar que pocas empresas, presentan adicional a sus estructuras propias, canales de distribución tercerizados. Además, la relación con estos canales es bastante fuerte por lo que difícilmente los nuevos competidores pueden acceder a ellos.

\section{g)Identificación de Marca}

Este factor puede convertirse en una barrera de entrada, en la medida que la imagen que tienen las empresas como consecuencia de su forma de actuar y las características del producto están influenciadas por la cultura y las preferencias del mercado local. De acuerdo a los resultados, el $86.67 \%$ considera que el conocer los gustos del mercado local, así como también la cultura de la ciudad son factores que se convierten en ventaja frente a posibles competidores. De esta forma se obtiene una fiabilidad que logra identificar los productos con la marca y fidelizar a los compradores.

En la mayoría de los casos, se manifestó que los principales compradores de cada una de las empresas encuestadas, valora el tiempo de la relación comercial al permitir que las empresas del sector puedan opinar e incluso sugerir nuevos productos o mejoras a los solicitados por sus compradores.

\section{h)Represalias}

Este factor se refiere a las acciones que toman las empresas ya existentes en el sector frente a la entrada de nuevos competidores. Las empresas encuestadas muestran que la principal acción implementada para contrarrestar a posibles entrantes consiste en la innovación (ver tabla 3), sin embargo, se deja claro que no basta con una sola estrategia competitiva, ya que en ocasiones deben tomar varias para afrontar esta situación.

Teniendo en cuenta que estas acciones requieren de distintos recursos, entre los cuales el económico toma especial relevancia, el $60 \%$ de las empresas señalan que no cuentan con recursos financieros para afrontarlas. Sin embargo, cuentan con cupos de crédito en entidades financieras las cuales podrían desembolsar el dinero en caso de ser necesario, aunque reconocen que estos siempre están supeditados a las condiciones del mercado y de la empresa, al momento del uso de los recursos de deuda.

\section{-ITabla 3.}

\section{Acciones frente a nuevos competidores}

Acciones Participación en respuestas (\%)

Innovación 40,91

Especialización 9,09

Capacitación 9,09

Importar recurso humano 4,55

Abrir nuevos mercados 4,55

Precio 4,55

Flexibilidad al cambio 4,55

Fidelización 4,55

Fuente: Elaboración propia.

\section{Fuerza 5: Amenazas de posibles sustitutos}

Los productos sustitutos deben suplir las mismas necesidades de los productos de la industria, para la comunicación gráfica en particular las tecnologías de la información y las comunicaciones pueden plantearse de acuerdo a la estrategia competitiva de la empresa como obstáculo u oportunidad de ventaja competitiva. En el caso de la industria de comunicación gráfica, el sustituto es aquel producto/ servicio que logre informar e inducir a un receptor a actuar de la manera en que el emisor está buscando. Para ello las tecnologías de la información y las comunicaciones (TIC) con herramientas electrónicas amigables al usuario y a través de las redes sociales como medio de difusión, han logrado dicho fin. Los emisores no buscan a las empresas para desarrollar la publicidad de su producto, sino que utilizan los medios existentes en las TIC para expresarlo, acá es discutible si esto pueda tener el mismo efecto que el trabajo profesional de los actores de la industria. 
La industria de la comunicación gráfica en Barranquilla, Colombia: Análisis sectorial desde el enfoque de las cinco fuerzas competitivas

La creciente disponibilidad de estos productos/servicios es preocupación permanente para los actores formales de la industria.

\section{a) Disponibilidad de sustitutos}

El $67 \%$ de las empresas encuestadas se sienten afectadas por las redes sociales que identifican como producto sustituto. Los empresarios indican que la alta informalidad del sector sobre todo en el área creativa (incluso más allá de las fronteras nacionales) ofrecen sus servicios a través de las redes sociales a precios muy bajos, lo que es una causante de afectación leve de momento, pero potencialmente grave a futuro. De la misma mane$\mathrm{ra}$, los empresarios advierten que sus productos y servicios son mucho más integrales y completos que los que se pueden encontrar en la franja informal de la industria. Adicionalmente, cabe anotar que los que no se sienten afectados expresan que el manejo de redes sociales, por el contrario, a lo expresado por los que se dicen afectados, se ha convertido con el pasar del tiempo en un valor agregado de sus servicios y en un componente estratégico del negocio, es decir lo incorporaron en su portafolio de productos.

\section{b)Acciones para enfrentar sustitutos}

En las entrevistas se logra establecer que la tendencia del negocio a la especialización y personalización ha dado paso a la aparición de las llamadas agencias de medios digitales, cuya misión es el manejo integral del negocio desde lo digital. Por lo tanto, la respuesta a este fenómeno en algunas empresas ha sido la creación de los departamentos de medios digitales que han sido los encargados de competir con estas nuevas agencias. Esto ha llevado a que los costos de nómina de las empresas formales se incrementen debido a que se ha contratado nuevo personal para potenciar la fuerza de las empresas desde esta nueva perspectiva. Cabe agregar que algunas empresas han venido implementando y ofreciendo el servicio de drones para publicitar productos y servicios de sus compradores.

El modelo de las cinco fuerzas competitivas permite precisamente revisar en la unidad de análisis de la industria aspectos relevantes también de su entorno. En la figura 6 se construye el modelo de las cinco fuerzas competitivas de la industria de la comunicación gráfica de la ciudad de Barranquilla. Allí se realiza un esbozo de los resultados obtenidos por medio de las encuestas, y se presentan los agentes económicos presentes en cada una de las fuerzas competitivas.

\section{Conclusión}

En lo que respecta a la fuerza "rivalidad" entre competidores, se destaca que hay una serie de empresas grandes, medianas y pequeñas que hacen que el mercado sea muy diverso en precios y calidad y que la oferta de productos sea compuesta básicamente por impresos y avisos con alto valor agregado, diferenciados y con tendencia a la personalización. Adicionalmente cabe anotar que los empresarios son categóricos en expresar que la industria se encuentra en crecimiento debido al auge económico que está viviendo la ciudad de Barranquilla. Esto indica que el ambiente competitivo del mercado es susceptible a la llegada de nuevos competidores y al aumento de capacidad productiva de las empresas apuntando al mejoramiento de su posición dentro del mercado y al incremento de sus márgenes de rentabilidad.

Por último, es de relevancia precisar que la estructura de costos de las empresas de la industria está compuesta mayoritariamente por costos fijos, sean estos de nómina o servicios públicos. Lo anterior desde el punto de vista financiero pone en cierto nivel de riesgo a estas empresas debido a que, de presentarse una ralentización o recesión de la economía, estos costos son más complicados en reducir. 
IFigura 6.

Modelo de las cinco fuerzas competitivas de la Industria Gráfica en Barranquilla

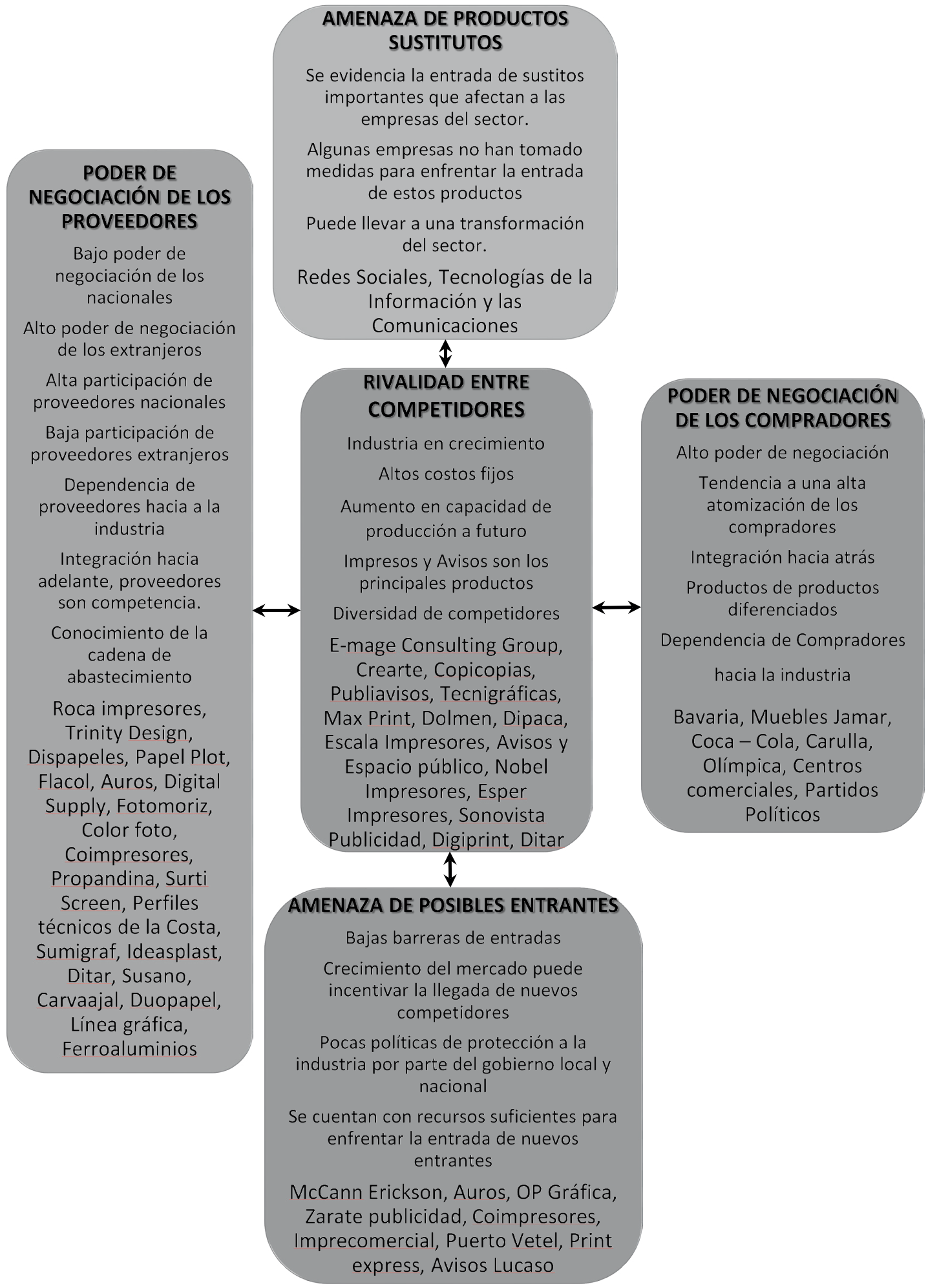

Fuente: Elaboración propia utilizando el modelo de Porter (2008) 
La industria de la comunicación gráfica en Barranquilla, Colombia: Análisis sectorial desde el enfoque de las cinco fuerzas competitivas

Del poder de negociación de proveedores se puede concluir, que el conocimiento de la cadena de abastecimiento por parte de las empresas de la industria, les permite tener una ventaja competitiva en el mercado, debido a que pueden planear su logística de abastecimiento y tener a su disposición la materia prima requerida para su operación. Así mismo, es importante mencionar que el poder de negociación de los proveedores nacionales de la industria es bajo, ya que las opciones son numerosas para las empresas de la industria que por algún motivo decidan cambiar de proveedor. Por el contrario, en el caso de los extranjeros se encuentra que estos tienen un alto poder de negociación con respecto a sus compradores nacionales debido a lo especializado de los productos y servicios que ofrecen. Mirando la participación de nacionales y extranjeros es necesario precisar que las empresas de la industria prefieren a los proveedores nacionales debido a que de esta manera no están expuestas a la volatilidad del dólar, situación a la que están enfrentadas las empresas que tienen proveedores en el exterior. Por último, no se puede desconocer que el $46 \%$ de los encuestados dijeron que algunos proveedores son exclusivos de la industria lo que podría llevar a una dependencia de estos hacia la industria lo que disminuiría un poco más su poder de negociación, adicionalmente se presenta una tendencia de integración hacia delante de los proveedores que conllevaría a que se conviertan en competencia de sus compradores con la presión resultante en la primera fuerza.

En el poder de negociación de compradores, la mayoría de las ventas de la industria se hacen de manera directa al consumidor final, solo en el $11.76 \%$ se utiliza intermediarios, esto significa que prácticamente no manejan canales de distribución, ellos mismos lo son. También es importante mencionar que en esta industria los compradores tienen un alto poder de negociación cuando se tienen en cuenta las numerosas empresas (agregando las micro e informales) que existen en la industria de la comunicación gráfica. El comprador tiene de donde escoger, si alguna empresa no cumple con los requisitos que este tenga con respecto a precio o calidad. Por otra parte, en la industria existe una cierta tendencia hacia la atomización de compradores que están en capacidad de comprar gran parte de la producción, esto influye en el nivel competitivo del mercado. La dependencia de los compradores hacia la industria es alta, en sectores particulares como: bebidas y alimentos, partidos políticos, eventos culturales y el sector comercial. Esta dependencia, según los encuestados, se da debido a que los ingresos y el éxito de las empresas de estos sectores depende en gran medida de la calidad de los productos que la industria gráfica ofrece: productos que son cada vez más diferenciados, con alto valor agregado y con tendencia a la personalización. Por último, existe una tendencia, aunque baja, de integración hacia cuando los compradores se convierten en competidores actuando como intermediarios entre las empresas y el consumidor final.

Como conclusión de la fuerza de posibles entrantes, se debe traer a colación el crecimiento de la industria como incentivo a una alta probabilidad de que empresas de fuera de la ciudad ingresen a este mercado. Es por esto que, si se quiere desincentivar la entrada de nuevos competidores, se sugiere fortalecer las barreras de entrada particularmente en los aspectos que dependen totalmente de las empresas: calidad, diferenciación, conocimiento del mercado y marca. Es sabido que se necesita un músculo financiero grande para entrar a la industria, lo que constituye per se una barrera de entrada, como también lo hace la curva de experiencia que utiliza el posicionamiento de la marca y fidelización de compradores. Adicionalmente hubo concordancia en todos los encuestados que su principal ventaja competitiva radica en el know how, representado en la creatividad y la innovación de sus productos, también consideraron como fortalezas: ser canales directos de distribución para no ceder parte de sus márgenes a los intermediarios, el conocer la cultura, gustos y preferencias del mercado local, ofreciendo a los compradores productos a la medida de sus necesidades y el atesoramiento de recursos para afrontar la entrada de nuevos competidores. Con respecto a las barreras gubernamentales, no existen políticas estructurales que protejan al sector, solo existe un beneficio arancelario puntual para importación de empaques plásticos, cuestión que no ayuda a la protección integral y por tanto no debe ser la opción del sector.

En definitiva, en la fuerza de los productos sustitutos aunque se ve un panorama de afectación con las tecnologías de la información y las comunicaciones. Los avances tecnológicos aunados a la concientización 
Jahir Enrique Lombana-Coy, Alberto Mario Molina-Rocha, Andrés Felipe Muñoz-Vergel y Jaime Alberto Muñoz-Vergel

ambiental en cuanto al uso del papel, invita a las empresas a diseñar estrategias competitivas destinadas a impedir la penetración de las empresas que vendan estos productos 0 implementar estrategias competitivas que permitan competir con ellas, de forma que integren estas nuevas tendencias al portfolio de sus empresas. Son las empresas que se han apropiado de las nuevas tecnologías en su interior las que las ven como oportunidades para abrir incluso nuevas líneas de negocio. En este sentido, también resulta importante mencionar, que actualmente, existe una tendencia alcista por el uso de redes sociales, las cuales, en muchas ocasiones, reemplaza los impresos, principales productos de la industria.

A pesar de que las condiciones coyunturales puedan ser complejas y adversas al entorno empresarial, las perspectivas de la industria en la región son positivas y de ahí que surja un interés adicional por continuar investigaciones alrededor de ella.

\section{Referencias Bibliográficas}

Asociación Colombiana de la Industria de la Comunicación Gráfica - ANDIGRAF, (2013-2015). Boletín Notigraf. Recuperado de: http://andigraf.com.co/boletin

Barney, J. B. (1991). Firm resources and sustained competitive advantage. Journal of Management, 17, 99-120.

Brandenburger A. \& Stuart, H. (1996), Value-based business strategy, Journal of Economics \& Management Strategy, (5), 5-24.

Brandenburger, A. (2002) Porter's Added Value: High Indeed! The Academy of Management Executive, 16, (2), 58-60

Departamento Administrativo Nacional de Estadística - DANE. (2013). Gran Encuesta Integrada de Hogares - GEIH. Recuperado de: http://formularios.dane.gov.co/ Anda_4_1/index.php/catalog/68/data_dictionary

Dunning, J. H. (1993). Internationalizing Porter's diamond. Management International Review, (33), 7-15.

Hoskisson, R. E., Eden, L., Lau, C. M. \& Wright, M. (2000). Strategy in emerging economies. Academy of Management Journal, (43), 249-67.

Mcgahan, A. M. \& Porter, M. (1997). How Much Does Industry Matter, Really? Strategic Management Journal, 18, (S1), 15-30.

Medina, A. F., Delgado, A. M. \& Lavado, P. A. (2012). Fundamentos de un sistema de gestión humana por competencias para soportar la estrategia organizacional en una pyme del sector de la industria de las artes gráficas en Cali (Colombia). Estudios Gerenciales, 28, (122). 121-138. Recuperado de: http://www.sciencedirect. com/science/article/pii/S0123592312701973

Ministerio de Comercio, Industria y Turismo. Programa de Transformación Productiva. (2008). Presente y Futuro del Sector. Recuperado de www.ptp.com.co/categoria/ industriaeditorial ycomunicaciongrafica.aspx

Ministerio de Comercio, Industria y Turismo - MinCIyT. (2012). Informe de Sostenibilidad Industria Editorial y de la Comunicación Gráfica. Programa de Transformación Productiva. Recuperado de http://www.ptp.com.co/ documentos/PTP_informe_sector_Editorial $\% 20$ y $\% 20$ de $\% 20$ la $\% 20$ Comunicaci $\%$ C $3 \%$ B 3n $\% 20$ Gr\%C3\%A1fica\%20\%20FINAL.pdf

Narayanan, V. K. and Fahey, L. (2005). The Relevance of the Institutional Underpinnings of Porter's Five Forces Framework to Emerging Economies: An Epistemological Analysis. Journal of Management Studies, 42 (1), 207223, Recuperado de http://ssrn.com/abstract $=648749$ Nelson, R. E. (1990). Is there strategy in Brazil? Business Horizons, July-August: 15-23.

Peng, M. W. \& Luo, Y. (2000). Managerial ties and firm performance in a transition economy: the nature of a micro-macro link. Academy of Management Journal, (43), 486-501.

Peteraf, M. A. (1993). The cornerstones of competitive advantage: A resource-based view. Strategic Management Journal. 14, (3), 179-191.

Porter, M. (1996) What Is Strategy? Harvard Business Review. November-December, 61-78.

Porter, M. (1979). How Competitive Forces Shape Strategy, Harvard Business Review. March-April, 86-93

Porter. M. (2008). The Five Competitive Forces that Shape Strategy, Harvard Business Review, January, 86-104

Rugman, A. M. \& Verbeke, A. (1993). Foreign subsidiaries and multinational strategic manage-ment: an extension and correction of Porter's single diamond framework. Management International Review, (33), 71-84.

Valero, E. A.. (2002). Clusters de empresas en la industria gráfica colombiana: dificultades y oportunidades. Innovar: Revista De Ciencias Administrativas y Sociales. Facultad de Ciencias Económicas, Universidad Nacional de Colombia, (19), 49-68. Recuperado de http://www.jstor.org/ stable/23741309.

Wernerfelt, B. (1984). A resource-based view of the firm, Strategic Management Journal, 5, 171-180.

Wright, M., Filatotchev, I., Hoskisson, R. E. \& Peng, M. W. (2005). Guest editor's introduction: Strategy research in emerging economies: Challenging the conventional wisdom'. Journal ofManagement Studies, 42 (1),1-33. 